\title{
Indoor Positioning Capabilities of Cognitive Entities
}

\author{
L. Kajdocsi \\ Széchenyi István University, Department of Information Technology \\ Egyetem tér 1, 9026 Győr, Hungary \\ e-mail: kajdocsi.laszlo@sze.hu
}

Abstract: Indoor Positioning is one of the most significant challenges of the $21^{\text {st }}$ century. Hence, this paper aims to give an overview on indoor navigation solutions and propose a theoretical hybrid method which is based on Bluetooth Smart technology and combination of distance measurement and pedestrian dead reckoning techniques. First of all, the paper presents radio based techniques. Secondly, other techniques based on the use of various sensors and measurements are described. Nowadays, it can be state that each technique requires any type of smart device such as smartphone or tablet, whose inertial sensors help in evaluation of pedestrians' actual position. Moreover, it would be assumed that each person has its own smart device, hence, it would be also state that they could be considered as one entity. Therefore, the researches on indoor navigation systems require novel low-cost approaches that involve new generation devices, thus create future networks.

Keywords: indoor positioning, indoor navigation system, indoor localization, smartphone

\section{Introduction}

Navigation was always an important part of people's everyday life. Therefore, it is hard to accomplish without a Global Positioning System (GPS) based navigation system in an outdoor environment today. Unfortunately, indoor positioning (IP) is still in its babyhood, although these services would be useful in many areas. Thus, indoor navigation would be crucial for logistics, industrial applications and for several consumer applications.

Several Indoor Positioning Systems (IPSs) have been developed over the last decade, relying on a wide variety of technologies, including radio, infrared and ultra-sound among others, but there are still few solutions available that are often expensive and complicated to establish. Some of existing indoor positioning proposals rely on a radio technology, e.g., Wi-Fi, Bluetooth (BT), or RFID. However, location sensing in indoor environments is a challenging task and an intensively researched issue. 
Recently, thanks to the technological advances of smartphones, IPSs have been designed to provide location information of persons and devices. Hence, smartphones have become crucial to indoor navigation. Moreover, a person and its smart device could be considered as one Cognitive Entity (CE). The term cognitive entity can describe any synergic combination of humans, devices, infrastructure and environment that is identifiable from the point of view of some cognitive capability [30]. CEs may have important role in navigation since they can be considered as the part of IP network. Thus, in positioning system the person's cognitive activity and state could be significant to its position too.

The main challenge of existing radio based proposals is the inaccuracy. Most of them use the Received Signal Strength Indicator (RSSI) measurement, the received Bit Error Rate (BER) or Cellular Signal Quality (CSQ) with triangulation or trilateration method. Distance estimation together with trilateration are one of the standard solutions for localization. But currently, the estimation accuracy is beyond the range of few meters. Although, a novel proposal use phase measurement for better distance estimation, which gives acceptable results [1].

Several proposals use Pedestrian Dead Reckoning (PDR) whose basis is to update the pedestrian's position by step length estimation using accelerometer and heading direction estimation using gyroscope and magnetometer. Although, the Earth's magnetic field is strongly perturbed inside buildings, thus making the measurement accurate for heading direction estimation is quite difficult. However, recent research gives an $80 \%$ improvement over PRD positioning using magnetometer measurement $[2]$.

Also often used technique is the Signal Strength (SS) fingerprinting, which compensates the drawback of the PDR more or less. The principle of this method is to measure several broadcaster's signal strength at runtime with mobile phone and compare the obtained data with the signal strength map generated earlier. To perform RSSI fingerprinting a database is needed that contains the signal strength information. This database gives the basis for comparison and then estimation. Common techniques for prediction would be one of the Nearest-Neighbor, Bayesian Filter, Particle Filter or Map Filtering algorithms or might be a combination of them [3, 4].

Besides the previously described radio based techniques, I have to mention several sensor based methods that are still too complicated or expensive to deploy. One of infrastructures is vision-based entity detection which would contain cameras of image sensors, infrared (IR) sensors or infrared Light Emitting Diodes (LEDs). IR sensors are widely used because they are low-cost components of vision-based indoor navigation [5]. However, most of them are strongly criticized as they disturb personal privacy. Another sensor based method uses ultrasonic where ultrasonic sensors measure distances by transmitting an ultrasonic signal and let it bounce back. It computes the distance from the duration taken by the signal to return to the receiver [6].

The paper is organized as follows. Section 2 presents the detailed principles of common IP techniques. Section 3 describes a novel proposed system and the related work. Finally, Section 4 concludes the paper and our future research direction. 


\section{State-of-the-art indoor localization methods}

In this section several trendy positioning methods from the past decade will be presented.

\subsection{Trilateration method}

The principle of trilateration approaches is that it requires at least three (in case of 2D) or four (in case of 3D) base transmitters with known coordinates. In case of four or more transmitters this method is called multilateration. If the distance from transmitter to $\mathrm{CE}$ could be measured, a circle can be drawn whose radius is the measured distance. The circles cross at a point which is the position of CE (Fig. 1). Nevertheless, the measured values are SSs rather than the distances. Although, the SS can be converted to a distance. The trilateration method is executed in two main steps. The first one converts the measured SS values to the distances. The other step uses any of geometric methods to evaluate the location.

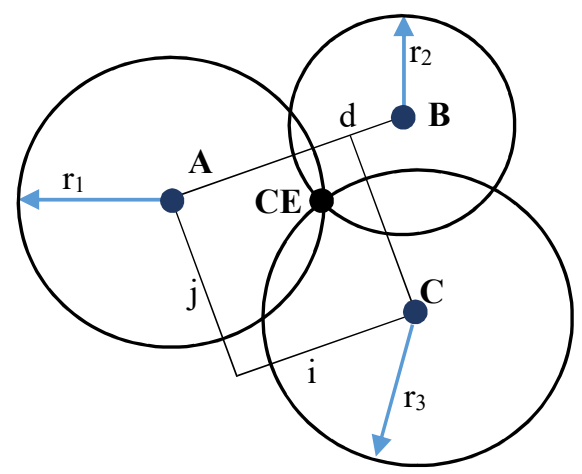

Figure 1. 2D trilateration

Assuming from Fig. 1 that the positions of transmitters on plane $z=0$ are $A(0,0)$, $B(d, 0)$ and $C(i, j)$ thus the position of $C E(x, y)$ can be computed as in (1) and (2).

$$
\begin{gathered}
x=\frac{r_{1}^{2}-r_{2}^{2}+d^{2}}{2 d}, \\
y=\frac{r_{1}^{2}-r_{3}^{2}+i^{2}+j^{2}}{2 j}-\frac{i}{j} x,
\end{gathered}
$$

Obtaining the distance measurement from the SS accurately is quite complex. The radio signal propagation is very complicated because of several influences in the environment. Thus, the signal strength weakens by the distance, the penetration losses through walls and floors and the other signal's interference is also a notable problem. Unfortunately, in the $2.4 \mathrm{GHz}$ band a lot type of devices can be the source of interference. In addition, the movement of people inside the building can considerably affect the SS. As the surroundings may differ from spot to spot, the addition of a new procedure is required for the trilateration method named learning procedure. 
In order to increase the trilateration's accuracy a hybrid method was proposed [7]. According to this method the propagation model behaves better in small localities. The principle of this method is to locate the small area where CE is and then using trilateration to compute the location of CE. This method notably improve the accuracy, but the trilateration approach is still worse than the other methods.

\subsection{Signal Strength Fingerprinting}

Fingerprinting method includes two main phases. One is offline (training) and the other is online (positioning). In the offline phase a fingerprint database is built and reference points have to be selected. To locate a $\mathrm{CE}$ at one reference point, all the transmitter's signal strengths must be measured. Thus, from measurements the main characteristic of the reference point is defined and is stored to the database. This step is repeated until all reference points are recorded. In the online phase CE measures the SS at a point where it is. The measured values are compared with the database's data applying a relevant matching algorithm.

There are two main approaches of fingerprinting that use pattern matching and/or searching technique: probabilistic method (neural networks, Bayesian-networks, etc.) and deterministic method (nearest-neighbor (NN), $K$-nearest-neighbor $(\mathrm{KNN})$, Weighted $K$-nearest-neighbor (KWNN)).

The easiest way of fingerprinting approach is deterministic one. Using this method, the construction of the database is nearly simple and the reference points are also easily determined by the average SSs of each transmitters. Several algorithms could be used to estimate the position of CE, but the basic is NN. From the point of view of indoor positioning, the $\mathrm{NN}$ tries to find a nearest signal distance. If $\mathrm{KNN}(\mathrm{K} \geq 2)$ is take into consideration, the average of the $\mathrm{K}$ coordinates is used to evaluate the position of $\mathrm{CE}$. In KWNN the weighting scheme is used, thus the weighted average is estimated rather than the average. Usually the KNN and the KWNN can perform better than the $\mathrm{NN}$ and their best result can be achieved when $\mathrm{K}$ equals 3 or 4 . However, when the density of reference points is high, the NN can perform as well as the more complicated methods. [8]

In general, probabilistic method uses one of Bayesian localizer (BL) or neural network. From experiments it can be stated that Bayesian approach performs higher accuracy than the nearest neighbor methods [4]. For positioning, the Bayes rule can be written as

$$
p(x \mid y)=\frac{p(y \mid x) p(x)}{p(y)},
$$

where $p(y \mid x)$ is the likelihood function, $p(x)$ is the prior probability and $p(y)$ is a normalizing constant. This probability of being at location $x$ is estimated for all fingerprints. Hence, the most probable location is the outcome of the BL.

The likelihood function can be estimated from the signal strengths. The frequency of each signal strength is applied to achieve a probability distribution. The Bayesian 
approach also estimates the prior probability $p(x)$ as the consistent distribution over all locations.

The other probabilistic technique uses neural networks during the offline phase. Here, the SS and the location coordinates are used as the inputs and the targets for the training goal. The input vector of SSs is multiplied by the trained input weight matrix and then added with input layer bias if bias is chosen. This result is put into the transfer function of the hidden layer neuron. The transfer function's output is multiplied by the trained hidden layer weight matrix and then added to the hidden layer bias if bias is chosen. Hence, the system's output is a two-element vector or three-element vector which is the result of the calculated 2D or 3D location. [9]

\subsection{Pedestrian Dead Reckoning (PDR)}

Pedestrian dead reckoning is a relative positioning technique based on low cost inertial sensors and transmitting devices. The current position of pedestrian can be estimated with the help of previews position $(\mathrm{X}(\mathrm{t}), \mathrm{Y}(\mathrm{t}))$, step length $(\mathrm{S})$ and heading direction $(\theta)$ as stated in (4) and (5).

$$
\begin{aligned}
& X(t+1)=X(t)+S \cdot \cos \theta, \\
& Y(t+1)=Y(t)+S \cdot \sin \theta,
\end{aligned}
$$

Since, the initial coordinates, the step length and the direction of movement are known the pedestrian's trajectory can be estimated. Usually, the step length can be estimated using accelerometer and the heading direction can be estimated with the help of magnetometer or gyroscope. The initial position is determined by the pedestrian's closeness to the transmitter. This closeness can be calculated from RSSI measurements.

Typically, two methods are used to obtain the displacement of pedestrian by using accelerometer signal: integration method and signal processing method. Applying the first method the displacement can be found by double integration of acceleration signal $[10,11]$. Nevertheless, the presence of noise in the accelerometer output causes a rapid growth of error. Besides, another source of error appears due to the gravity of the earth when the phone has a random orientation.

The second method of obtaining the linear movement is the signal processing [12, 13]. First of all, in a smartphone based PDR system the activity classification is the initial procedure, i.e., the pedestrian state is recognized whether it is static or walking. In this method the foot step detection has to be performed by analyzing the accelerometer signals. In addition, the pedestrian's linear movement is calculated using stride length estimation methods. In the end, the heading direction is determined using magnetometer or gyroscope data.

Typically, there are three types of step detection that use accelerometer data $[14,15]$ :

- $\quad$ peak detection

- $\quad$ zero crossing detection

- flat-zone detection 
While, there are other approaches utilized for step detection more or less expansively [16]:

- autocorrelation

- Fast Fourier Transformation (FFT)

- $\quad$ stance-phase detection

The peak detection is not really suitable for step detection because the accelerometer's output is significantly influenced by the pedestrian's walking velocity [17]. The drawback of the flat zone method is that the signal is not detected when the accelerometer is attached to the pedestrian's waist [15]. Thus, the zero-crossing method seems to be the most applicable as it is resilient to the pedestrians walking velocity [18].

Once the steps are detected, the step length is still required to calculate. According to Groves proposition [19] a method to estimate the step length is to presume that each steps have equal size. Unfortunately, this presumption is not always true because the step length is not a constant value. There are still some different methods for stride length estimation where the acceleration sensor is attached to the pedestrian's foot $[10$, 14]:

- Weinberg approach [20]

- Scarlet approach [21]

- Kim approach [13]

The last part of PDR positioning is to estimate the pedestrian's heading direction. Magnetometer and gyroscope are widely used to calculate the heading. The magnetometer can be used to determine the pedestrian's initial absolute heading. While, the gyroscope is used to estimate the change in pedestrian's relative heading. Therefore, the combination of two sensors could be used in a hybrid system.

According to [2], a new method utilises two components in order to pre-filter perturbed

measurements and fuse the data collected by multiple users to improve the heading estimate accuracy. It can be also stated that the use of one of the two components leads to an insufficient improvement in PDR localisation. In fact the proposed system by [2] reaches an error reduction of $83.7 \%$ in the heading estimation and localisation error reduction of $80 \%$.

\subsection{Vision-based positioning}

Visible light communication (VLC) is a remarkable technology for indoor positioning. VLC can be used in environments where radio frequency is constrained. Recently, the Light Emitting Diode (LED) is more advantageous than regular lighting devices. Hence, LEDs are strong candidates for VLC based IPSs no matter if it is white light LED of infrared light LED. Typically, visible light positioning (VLP) uses image sensors. This approach applies LED array as the transmitter of 3D coordination information of reference LEDs. Sensors receive the information from all reference transmitters and demodulate the position information. The required position is then 
estimated using the positions of reference LEDs and the geometric relationship of images on the sensors. In this solution at least four LEDs from the array transmit their 3D position information to the image sensors. The chosen LEDs must not be collinear. To estimate the unknown position the most commonly used method is the least square estimation (LSE). The main drawback of the VLP is that natural and artificial light can interfere with LED light which could strongly increase the inaccuracy of the method. Besides, vision-based localization systems are widely criticized because they disturb personal privacy.

\subsection{Sound-based positioning}

The principle of this technique is to use emitters that transmit ultrasonic waves with a short wavelength. In dry air at a temperature of $25^{\circ} \mathrm{C}$ the speed of sound is approximately $346 \mathrm{~m} / \mathrm{s}$ which is much lower than the speed of light. Therefore, it is possible to work with sound with a fine accuracy. To determine the location using ultrasonic waves the time of arrival (TOA) or the time difference of arrival (TDOA) method can be used. Several well-known ultrasonic positioning systems are Bat [22], Constellation [23], Cricket [24, 25], Dolphin [26], Buzz [27], Walrus [28] and BeepBeep [29].

Currently, ultrasonic positioning is the most accurate approach for indoor localization. It easily passes the one meter barrier and comes very close to the one centimetre accuracy. The main drawback of ultrasonic positioning systems is the expensiveness of deployment because they require special electronics that are not implemented into consumer smartphones.

\section{Proposed work}

Several approaches are describes in Section 2 related to indoor localization. Some of them are less accurate but some are mostly accurate. Selection of the perfect method is very complex because there are several aspects that have to be taken into consideration. These are the accuracy, the cost effectiveness, the complexity of hardware design and the computational requirements.

Numerous researches work on the solution of indoor localization but there is still an open challenge in deployment of a low cost consumer IPS. Applying smartphones in localization is not a novel issue but the methods that utilize the phone's built-in sensors are mostly inaccurate. However, designing of separate circuits with more accurate sensors is too expensive for consumer use.

During my literature research it is realized that a cost effective and low energy solution is needed that has low computational requirement. Luckily, the technological advance of smart phones rises quickly, hence, even the budget phones run quad-core or octa-core processors that push the computational capacity. Traditionally, nowadays phones come with gyro sensor, acceleration sensor, magneto sensor, gravity sensor, WiFi and Bluetooth.

In this section a theoretical hybrid system is proposed that will combine distance estimation and pedestrian dead reckoning methods. Bluetooth low energy (BLE) beacons are selected as transmitters of reference coordinates and a consumer 
smartphone must be chosen with inbuilt accelerometer, gyroscope and magnetometer. To reduce the computational necessity of the smart device a hypothesis must be satisfied which assumes that beacons need to be communicate with each other in a full or partial mesh network topology. Mesh technology is regarded as a key to many Internet of Things (IoT) applications, especially those that require peer-to-peer communication. Moreover, beacons would perform some computations and share the calculated results with the other beacons and CEs. The proposed idea is represented in Fig. 2.

Currently, the consumer smart devices such as smartphones and tablets support Bluetooth 4.0 specification. Unfortunately, BT $4.0 \mathrm{spec}$ does not allow mesh technology yet [31]. This issue is also a great challenge in networking and BT-based technologies.

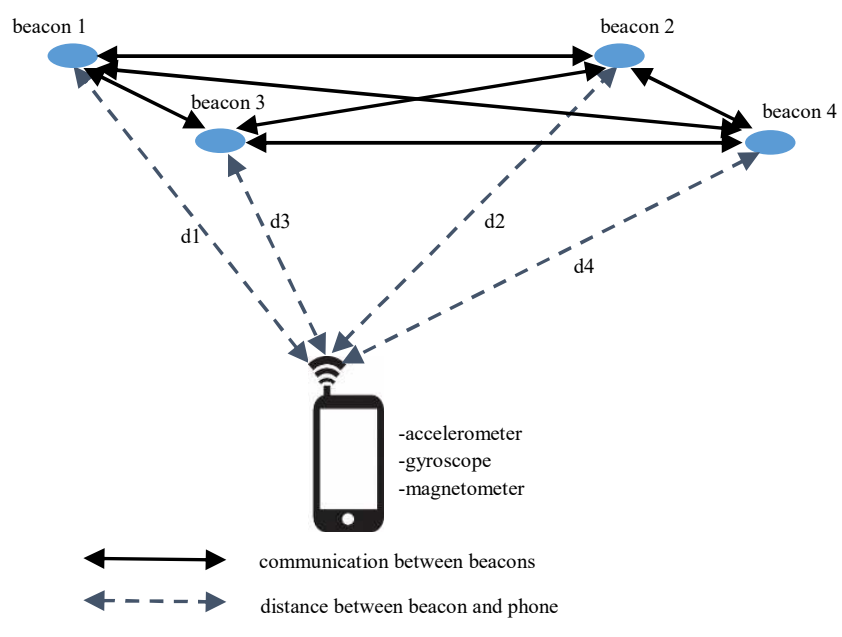

Figure 2. Basic structure of hybrid positioning system based on pedestrian dead reckoning, distance measurement and mesh technology

However, in 2015 the Bluetooth Special Interest Group (SIG) officially announced the formation of the Bluetooth Smart Mesh Working Group (SMWG). This working group have built the architecture for standardized mesh networking capability for Bluetooth Smart technology. The SIG will look to officially adopt profiles this year. Moreover, SMWG's goal is to have new mesh profiles run not only on the new BT 4.2 spec, but also on the legacy BT 4.0 spec.

After all, BT $4.0 \mathrm{spec}$ still does not support mesh technology on profile layer. However, a quasi-mesh communication would be accomplished on hardware level. Assuming that two BLE processors are built in one Printed Circuit Board (PCB) from which either runs in peripheral role and the other in central role. The data exchange between the central and the peripheral processor can be performed via serial communication interface. Peripheral devices are resource constrained devices that can connect to a much more powerful central device. Central device is usually the cellphone or tablet that the peripheral connect to with far more processing power and memory. 
Hence, the smart devices can connect to any peripheral and each peripheral is able to communicate with its central neighbor. While, any central can connect to any peripheral. Besides, a peripheral can connect to more centrals at a time.

The drawback of this structure may be the loss of its online behavior, because of discontinuous communication. One central device is able to connect to one peripheral at a time through RF band, but meanwhile it can communicate with its peripheral neighbor via serial interface. The main benefit of the proposed system may be faster computation process and thus, a more accurate system would be developed.

Currently, there are plans to implement the proposed approach in the near future. DA14580 SmartBond beacons will be deployed as nodes of the mesh network. Furthermore, the development of Android and iOS applications is needed as well. These applications will process the data between beacons and phones and assist in testing mesh network features.

\section{Conclusion}

The current overview presents the trendiest methods of indoor positioning such as trilateration based on distance estimation, fingerprinting based on a matching algorithm, pedestrian dead reckoning based on step length and heading direction estimations, vision- based positioning and sound-based positioning.

In Section 2.1 a hybrid method was mentioned according to [7] which significantly increases the accuracy of trilateration but does not make it better than other methods. Section 2.2 introduces the two main fingerprinting approaches that are deterministic and probabilistic ones. This method is more accurate than the previews one, but it requires remarkable computational capacity. In Section 2.3 Pedestrian Dead Reckoning is presented that is based on step length calculation from acceleration signal and heading estimation using gyroscope and magnetometer. A proposed work by [2] gives a localization error reduction of $80 \%$. Section 2.4 describes that visible light communication is an impressive technology for indoor positioning which can produce appropriate accuracy in localization estimation. The drawback of this solution is rather a legal issue than technical, because it disturbs personal privacy. Lastly, Section 2.5 represents ultrasonic positioning that is highly the most accurate technique among indoor localization methods, but the expensiveness of deployment prevents the widespread application.

Summarizing all the aforementioned information a low-cost and low-energy solution is needed in localization. BLE devices and consumer smart devices satisfy these criteria. The smart devices' built-in low-cost sensors (accelerometer, magnetometer and gyroscope) are responsible for pedestrian dead reckoning process which calculates CE's position using step length and heading estimations. If BLE beacons could communicate in mesh network that would notably speed up the data exchange and the computing process between beacons and smart devices. As a result of such a structure an effective positioning system can be developed for indoor environments. The future of work is to install the beacons in an indoor environment and to design the algorithm of the proposed hybrid method. 


\section{References}

[1] Pelka M, Bollmeyer C and Hellbruck H: Accurate Radio Distance Estimation by Phase Measurements with Multiple Frequencies. In Proceedings of International Conference on Indoor Positioning and Indoor Navigation (IPIN2014), pp. 142151, Busan, 2014 DOI: $10.1109 /$ IPIN.2014.7275478

[2] Abadi M J, et al.: A Collaborative Approach to Heading Estimation for Smartphone-based PDR Indoor Localisation. In Proceedings of International Conference on Indoor Positioning and Indoor Navigation (IPIN2014), pp. 554563, Busan, 2014 DOI: $10.1109 /$ IPIN.2014.7275528

[3] Widyawan, Klepal M and Pesch D: Influence of Predicted and Measured Fingerprint on the Accuracy of RSSI-based Indoor Location Systems. In Proceedings of the $4^{\text {th }}$ Workshop on Positioning, Navigation and Communication, pp. 145-151, Hannover, 2007

DOI: 10.1109/WPNC.2007.353626

[4] Honkavirta V et al.: A Comparative Survey of WLAN Location Fingerprinting Methods. In Proceedings of the 6th Workshop on Positioning, Navigation and Communication, pp. 243-251, Hannover, 2009 DOI: $10.1109 /$ WPNC.2009.4907834

[5] Jovanovic N, Ozcelebi T and Lukkien J: Indoor User Positioning using Infrared LEDs and Sensors. In Proceedings of International Conference on Indoor Positioning and Indoor Navigation (IPIN2014), pp. 400-406, Busan, 2014 DOI: $\underline{10.1109 / \text { IPIN.2014.7275508 }}$

[6] Png L C, Chen L, Liu S and Peh W K: An Arduino-based Indoor Positioning System (IPS) using Visible Light Communication and Ultrasound. In Proceedings of International Conference Consumer Electronics - Taiwan (ICCE-TW), pp. 217218, Taipei, 2014 DOI: $10.1109 /$ ICCE-TW.2014.6904066

[7] Li B, Dempster A, Rizos C and Barnes J: Hybrid Method for Localization Using WLAN. In national biennial Conference of the Spatial Sciences Institute, Melbourne, 2005

[8] Li B, Salter J, Dempster A G and Rizos C: Indoor Positioning Techniques Based on Wireless LAN. In Proceedings of the 1st IEEE International Conference on Wireless Broadband and Ultra Wideband Communications, pp. 130 - 136, 2006

[9] Liu H, Darabi H, Banerjee P and Liu J: Survey of Wireless Indoor Positioning Techniques and Systems. In Proceedings of IEEE Transactions on Systems, Man, and Cybernetics, Part C: Applications and Reviews, Vol. 37, No. 6, pp. 10671080,2007 DOI: $10.1109 / \mathrm{TSMCC} .2007 .905750$

[10] Jimenez A R, Seco F, Prieto C and Guevara J: A Comparison of Pedestrian DeadReckoning Algorithms using a Low-Cost MEMS IMU. In Proceedings of IEEE International Symposium on Intelligent Signal Processing (WISP2009), pp. 37-42, Budapest, 2009

DOI: $10.1109 /$ WISP.2009.5286542 
[11] Skog I, Nillson J-O and Händel P: Evaluation of Zero-Velocity Detectors for Foot-Mounted Inertial Navigation Systems. In Proceedings of International Conference on Indoor Positioning and Indoor Navigation (IPIN2010), pp. 1-6, Zurich, 2010

DOI: $10.1109 /$ IPIN.2010.5646936

[12] Feliz R, Zalama E and Gómez J: Pedestrian tracking using inertial sensors. Journal of Physical Agents, Vol. 3. No. 1, pp. 35-42, 2009

[13] Kim J W, Jang H J, Hwang D-H and Park C: A Step, Stride and Heading Determination for the Pedestrian Navigation System. Journal of Global Positioning Systems, Vol. 3 No. 1-2, pp. 273-279, 2004

[14] Gupta S K, Box S and Wilson R E: Low cost infrastructure free form of indoor positioning. In Proceedings of International Conference on Indoor Positioning and Indoor Navigation (IPIN2014), pp. 11-18, Busan, 2014

DOI: $10.1109 /$ IPIN.2014.7275462

[15] Shin S H et al.: Adaptive Step Length Estimation Algorithm Using Low-Cost MEMS Inertial Sensors. In Proceedings of IEEE Sensors Applications Symposium, pp. 1-5, San Diego, CA, USA, 2007 DOI: $10.1109 /$ SAS.2007.374406

[16] Chen R, Pei L and Chen Y: A Smart Phone Based PDR Solution for Indoor Navigation. In Proceedings of the $24^{\text {th }}$ International Technical meeting of the Satellite Division of The Institute of Navigation, pp. 1404-1408, Portland, OR, USA, 2011

[17] Ali A and El-Sheimy N: Low-Cost MEMS-Based Pedestrian Navigation Technique for GPS-Denied Areas. Journal of Sensors, Vol. 2013, Article ID 197090, pp. 1-10, 2013

[18] Beauregard S and Haas H: Pedestrian Dead Reckoning: A Basis for Personal Positioning. In Proceedings of the 3rd Workshop on Positioning, Navigation and Communication (WPNC'06), pp. 27-35, Dresden, 2010

[19] Budiyono A: Principles of GNSS, Inertial, and Multisensor Integrated Navigation Systems. Book review from Industrial Robot: An International Journal, Vol. 39, No. 3, 2012

[20] Weinberg H: Using the ADXL202 in Pedometer and Personal Navigation Applications. In Application Notes American Devices; Analog Devices, Inc.: Norwood, MA, USA, 2002

[21] Scarlett J: Enhancing the Performance of Pedometers Using a Single Accelerometer. Analog Devices, 2007

[22] Ward A, Jones A and Hopper A: A New Location Technique for the Active Office. Journal of Personal Communication, Vol. 4, No. 5, pp. 42-47, 1997 DOI: $10.1109 / 98.626982$

[23] Foxlin E, Harrington M and Pfeifer G: Constellation - A wide-range wireless motion-tracking system for augmented reality and virtual set application. In Proceedings of the 25th annual conference on Computer graphics and interactive techniques, pp. 371-378, New York, NY, USA, 1998

DOI: $10.1145 / 280814.280937$

[24] Priyantha N B, Chakraborty A and Balakrishnan H: The Cricket Location-Support System. In Proceedings of the $6^{\text {th }}$ Annual ACM International Conference on Mobile Computing and Networking (MOBICOM), pp. 32-43, Boston, MA, USA, 
2000

DOI: $\underline{10.1145 / 345910.345917}$

[25] Balakrishnan H and Priyantha N: The Cricket Indoor Location System: Experience and Status. In Proceedings of the 2003 Workshop on Location-Aware Computing, pp. 7-9, Seattle, Washington, USA, 2003

[26] Hazas M and Ward A: A Novel Broadband Ultrasonic Location System. Ubiquitous Computing: 4th International Conference Göteborg, Vol. 2498 of the series Lecture Notes in Computer Science, pp. 264-280, Göteborg, 2002 DOI: $10.1007 / 3-540-45809-321$

[27] Mccarthy M R: The BUZZ: Narrowband ultrasonic positioning for wearable computers. Ph.D dissertation, University of Bristol, Faculty of Engineering, Department of Computer Science, 2007

[28] Borriello G et al.: WALRUS: Wireless Acoustic Location with Room-Level Resolution using Ultrasound. In Proceedings of the 3rd International Conference on Mobile Systems, Applications, and Services, Applications, and Services, pp. 191-204, Seattle, WA, USA, 2005

[29] Peng C et al.: BeepBeep: A High Accuracy Acoustic Ranging System using COTS Mobile Devices. In Proceedings of the 5th international conference on Embedded networked sensor systems, pp. 1-14, New York, NY, USA, 2007 DOI: $10.1145 / 1322263.1322265$

[30] Baranyi P, Csapo A and Sallai Gy: Cognitive Infocommunications (CogInfoCom). Springer International Publishing, Switzerland. 2015 DOI: 10.1007/978-3-319-19608-4

[31] "Bluetooth Core Specification Version 4.0", 2010. Available at: https://www.bluetooth.com/specifications/adopted-specifications 